\title{
Intelligent Approaches to Real Time Level Control
}

\author{
Snejana Yordanova \\ Technical University of Sofia, Faculty of Automation, Sofia 1000, Bulgaria \\ E-mail: sty@tu-sofia.bg
}

\begin{abstract}
Liquid level control is important for ensuring energy and material balance in many installations but it also difficult as the plant is nonlinear, inertial and with model uncertainties. Fuzzy logic controllers (FLCs) are successfully applied to ensure system stability and robustness by simple means and a model-free design. This paper suggests a procedure for off-line tuning of the many FLC parameters based on optimization of a suggested multi-objective function defined on several system performance indices using genetic algorithms (GAs). First, a model-free FLC is empirically tuned, then applied for real time control of the plant and the necessary data recorded and used to GA parameter optimize a TSK plant model of an accepted structure. The validated on different set of experimental data model is employed in FLC closed loop system simulation experiments to evaluate the fitness function in the GA optimization of the FLC pre-processing and postprocessing parameters. The procedure is applied for the real time PI/PID FLC level control in a laboratory-scale tank system. The improvement of the system performance indices due to the GA optimization is estimated in level real time control.
\end{abstract}

Index Terms-Fuzzy logic level control, genetic algorithms, multi-objective optimization, real time, TSK plant modeling.

\section{INTRODUCTION AND RELATED WORK}

Liquid level control is important for ensuring energy and material balance in most installations such as boilers, evaporators, reactors, distillation columns, etc. [1-3]. The plant is nonlinear, inertial and with model uncertainty. Fuzzy logic controllers (FLCs) are successfully applied to guarantee system stability, good performance and robustness for different operation points and disturbances. They are favoured also for their simple structure and design, based only on expert information about the plant [4]. A FLC for level control by changing the pump flow rate on the basis of a Takagi-Sugeno-Kang (TSK) dynamic plant model and the principle of parallel distributed compensation (PDC) of the local linear plants is designed in [5]. In [6-10] various FLCs for level control are developed using two (system error and rate of error) or three (level, flow rate and pressure) input variables and one (valve opening) or three (valve opening, fuel and steam flow rate) output variables.
The great number of FLC's parameters - scaling factors (ScFs), number, type and parameters of the membership functions (MFs), rule base, parameters of the pre- and post-processing, etc. are empirically tuned. In order to put the FLC tuning on objective grounds a multiobjective nonlinear optimization technique is often applied optimizing various system performance and energy efficiency indices under a constrained control. The optimization procedure is usually carried out off-line not to interfere in the real time plant control and is based on existing analytical or simulation nonlinear plant model.

There is a variety of optimization methods derivative-based or derivative-free, stochastic or deterministic. The genetic algorithms (GAs) as a derivative-free, stochastic and parallel search technique for multi-objective global optimization of a nonanalytically and often experimentally defined multimodal nonlinear function of many variables (parameters) with or without constraints are considered suitable for FLC tuning [11]. The GA optimization is difficult to apply on-line as it interferes the plant operation, is slow a great number of experiments are required, is inaccurate because of the many disturbances from the industrial environment, and is restricted by the system stability and parameter and signal constraints. The off-line GA optimization is based on an accurate plant model, an accepted fitness function and a representative sample of experiments/simulations used for its evaluation.

There are various applications of GAs for tuning of level controllers. Linear PID for level control is GA tuned in coupled tanks in [12] where the improvement of the performance of the PID control system compared to the ordinary tuned PID system is shown in simulations. In [13] the PID level controller is on-line GA optimized during the real time control which results in many trial experiments and limitations related with ensuring of system stability which points out the advantages of an off-line GA optimization. GA is used in [9] for reduction of the number of rules in the design of a FLC for the level control in a boiler. Simulations and a comparison with a linear PID controlled system prove a decrease of overshoot $\sigma$ and settling time $t_{\mathrm{s}}$. GA tuning of 39 parameters - the bounds for the MFs and the singletons in the fuzzy rules conclusions of a FLC for the control of the liquid level in a tank is suggested and tested in real time control in [14] using an artificial neural network (ANN) plant model. The fitness function is based on the error between actual and desired trajectory. A decrease of the 
closed loop FLC system $t_{\mathrm{s}}$ and $\sigma$ has been proven. In [15] GAs are applied to off-line tune a neuro-fuzzy controller for a laboratory-scale coupled-tank liquid-level real time control using a linear plant model identification. A PIDFLC has been designed in [16] reducing the number of rules and optimizing the parameters of the MFs by GA technique with several fitness functions and combinations of them. Simulations show decreased $t_{\mathrm{s}}$ and $\sigma$ of the system response and also smooth control and reduction of energy consumption when compared to linear PID system or PID-FLC system designed empirically. In [17] FLC with GA optimized MFs widths and ScFs is applied to both - water/steam temperature and water level control in a fire tube boiler and the system performance improved compared with the FLC system. In [18] the merits of a FLC with respect to a conventional controller are outlined in maintaining a desired cane level in chute during juice extraction at different references.

The common drawbacks of these FLC-GA based approaches to level control can be summarized as follows:

- the plant model used in the off-line GA optimization of the FLC is either a simple linear model, derived via identification in a single operation point, or analytically derived, or an existing TSK model, or difficult to derive (a complicated ANN which is trained and validated from long and hard for the plant experiments);

- the restrictions related to preservation of system stability in the on-line GA optimization of the FLC in real time control lead to an inaccurate tuning and a long experimentation during which the system performance is worsen;

- the performance improvement of the designed FLCGA system is demonstrated mainly in simulations, a more realistic approach is to use a pilot plant and real time control in an environment more close to the industrial;

- a great number of parameters is optimized instead of selecting a few most effective;

- improper fitness functions are selected (some integral system performance measure such as integral time squared error -ITSE, deviation from designed trajectory, etc.) which are complex, depend on the experiments sample and do not take advantage of the GAs facilities for a multi-objective optimization by combining different requirements [14].

The aim of the present work is to develop a procedure for off-line GA optimization of the parameters of the preand post-processing of a model-free PI/PID-FLC on the basis of real time level control data and a suggested multi-objective fitness function and to compare the performances of the closed loop systems in real time control of FLC systems with empirical and GA optimized FLC tuning. The PI/PID-FLC is with a widely spread structure of a fuzzy unit (FU) with normalized inputs the system error $e$ and derivative-of-error $\dot{e}$, and an output - the rate of control $d u$, and the post-processing is integral for a PI-FLC and proportional-plus-integral (PI) for a PID-FLC respectively.

Manuscript received May 27, 2015; revised June 25, 2015; accepted June 30, 2015.
The plant is a laboratory-scale tank level control system equipped with industrial measuring transducers and actuators and a MATLAB ${ }^{\mathrm{TM}}$ real time controller. The novelty concludes in the use of a TSK plant model in the off-line GA optimization of the FLC, derived via GA parameter optimization of an accepted structure using experimental data from the real time level control with an empirically designed model-free FLC. The suggested objective (fitness) functions in the TSK plant modeling and the FLC tuning are related to the high accuracy of the TSK plant model and the desired performance of the FLC closed loop system which combines several performance measures respectively. The design, the simulation and the real time investigations are based on MATLAB ${ }^{\mathrm{TM}}[19$, $20]$.

The paper is organized in the following manner. In Chapter 2 the previous related investigations and the problem formulation are presented. A TSK plant model is derived in Chapter 3 via GA optimization using experimental data from closed loop level control in real time with empirically tuned PI/PID-FLC. The procedure for off-line GA optimization of the FLC tuning parameters based on a suggested multi-objective fitness function and simulation investigations of the closed loop system with the derived TSK plant model is explained in Chapter 4. In Chapter 5 the results from the real time level control with the empirically and the GA tuned FLCs are described and discussed. Chapter 6 summarizes the main contributions and outlines the future research.

\section{Previous InVESTigations AND PRoblem FORMULATION}

The system to be designed consists of a PI/PID-FLC, built on a two-input FU with normalized inputs $e$ and $\dot{e}$ and output $d u$ and pre- and post-processing, and a nonlinear plant. The PI/PID-FLC parameters $\mathbf{q}_{\text {PI/PID-FLC }}$ to be GA optimized include: 1) the ScFs for normalization of the error $K_{\mathrm{e}}$ and the derivative of error $K_{\mathrm{de}}$ and for denormalization of the derivative of the control $K_{\mathrm{du}} ; 2$ ) the parameters of the commonly used first order differentiator for computing of $\dot{e}$ with transfer function $W_{\mathrm{d}}(s)=K_{\mathrm{d}} \cdot T_{\mathrm{d}} \cdot s /\left(T_{\mathrm{d}} \cdot s+1\right)$; 3) parameters of the postprocessing integrator or of the PI algorithm $W_{\mathrm{PI}}(\mathrm{s})=K_{\mathrm{p}}+K_{\mathrm{i}} / \mathrm{s}$, which are united with $K_{\mathrm{du}}$. Thus the tuning parameters of the PI/PID-FLC are $\mathbf{q}_{\text {PI/PID-FLC }}=\left[K_{\mathrm{e}}\right.$ $K_{\mathrm{de}} K_{\mathrm{d}} T_{\mathrm{d}} K_{\mathrm{du}}$ or $\left.\left(K_{\mathrm{p}} K_{\mathrm{i}}\right)\right]$.

The present work is based on the developed design procedures for off-line GA tuning of linear controllers [21] and local linear controllers in a PDC used with a FL supervisor (FLS) [22] applied for temperature real time control. There it is established in real time control that the effect of GA optimization of the main linear controller is equivalent to using of an on-line FL supervisor. The two techniques complement when the FLS is built on different system performance measures from the used in the GA optimization fitness function, e.g. the settling time $t_{\mathrm{s}}$ and the overshoot $\sigma$ are reduced due to GA optimization of the main controller and the control effort $u$ is reduced due to the connecting of the GA optimized 
FLS [22].

In [23] a PI-FLC, a PID-FLC and linear PI and PID controllers are designed for level control and their tuning parameters - the parameters of the pre- and post processing, are GA optimized on the basis of an analytical plant model, simulations and a fitness function that combines of integral square relative error with respect to reference $y_{\mathrm{r}}$, integral square relative control action with respect to maximal control $u_{\max }$ and an estimate $\max \left|e_{\min }(t) / y_{r}\right|$ for the maximal overshoot

$\sigma=y / y_{\mathrm{r}}-1$. The GA designed systems have reduced $t_{\mathrm{s}}$, $\sigma$ and control effort estimated via simulations in comparison to systems with empirically tuned controllers.

In this investigation the GA tuning of the PI/PID-FLC pre- and post-processing parameters is based on the same fitness function, suggested in [23]:

$$
\mathbf{F}=\int\left[e(t) / y_{\mathrm{r}}\right]^{2} d t+\int\left[u(t) / u_{\max }\right]^{2} d t++\max \left|e_{\min }(t) / y_{\mathrm{r}}\right| \rightarrow \min _{\mathrm{q}_{\text {PripD D.tC }}}
$$

The necessary for the off-line GA PI/PID-FLC tuning nonlinear plant model that is used to simulate the closed loop system responses to step references and disturbances with various magnitudes and frequencies in order to compute (1) for the current PI/PID-FLC parameters (chromosome), is the suggested in [19] TSK plant model. It is comprises a Sugeno model and parallel suitable linear dynamic models to represent the local linear models dynamics. The novelty in the present approach is: 1) the GA optimization of the TSK plant model parameters of both the dynamic models and the Gaussian membership functions of the Sugeno model using the same fitness function - the integral square relative error between real plant output $y$ and TSK model output $y_{\mathrm{TSK}} ; 2$ ) the data sample for the plant output and input are recorded from the plant real time control in a closed loop system by an empirically tuned model-free PI/PID-FLC, so that these data contain rich in magnitudes and frequencies signals from the real time operation range necessary for modeling a nonlinear plant; 3 ) the less rich and proper data from a restricted identification are used for validation of the derived TSK plant model; 4) the designed system performance improvement in sense of reduced settling time, overshoot and control effort and increased system robustness and control smoothness is assessed in real time control on a pilot plant in environment close to the industrial via comparison of the GA optimized FLC system and the empirically tuned FLC system.

The main problems to be solved in the present investigation are:

1. Derivation of a TSK plant model via GA optimization of the parameters of an accepted structure MFs and dynamic models parameters, and model validation using data from level real time control on the basis of empirically designed model-free PI/PI-FLC.

2. GA optimization of the parameters of the pre- and post-processing of the PI/PID-FLC using the multiobjective fitness function (1) and simulations of the level control system with the derived TSK plant model.

3. Real time control of level with GA optimized and with empirically tuned PI/PID-FLC and estimation of improvements by comparison of the two control systems performances - settling time, overshoot, control effort and smoothness, system robustness, etc.

\section{TSK PLANT MODELING}

The laboratory-scale pilot plant, in which the level $H$ of liquid is to be controlled by the flow rate of a pump via a controller, embedded on a computer in a Simulink model, is shown in Fig. 1. It consists of a tank, a collective tank, a sunk level transducer on the basis of a differential pressure cell for measuring of level $H$ in the range $[0.3,50], \mathrm{cm}$ with normalized output $[0,10], \mathrm{V}$, connected to an analog-to-digital converter (ADC) on a data acquisition board (DAQ), a tap for manual fixing of the tank outflow flow rate, a DC pump for input $[0,12]$, $\mathrm{V}$, controlled by the Simulink controller via a digital-toanalog converter (DAC) on the DAQ and a pump power amplifier. At each sample time $t_{\mathrm{k}}$ the measured level signal at the output of the level transducer is passed to the ADC of the DAQ board and read by the Analog Input (the corresponding driver) block in the Simulink model. There, it is converted into level $H_{\mathrm{k}}$, plotted via the graphrecorder, compared with the current reference $H_{\mathrm{rk}}$ and the error $e_{\mathrm{k}}$ is processed by the Simulink blocks that comprise the controller to compute the corresponding control action $u_{\mathrm{k}}$, which is limited in the range $[0,10]$ and via Analog Output (driver) block passed onto DAC and the pump power amplifier, connected to the pump input. Thus the flow rate of the inflow liquid to the tank is changed by pumping into the tank more or less of the liquid from the collective tank.

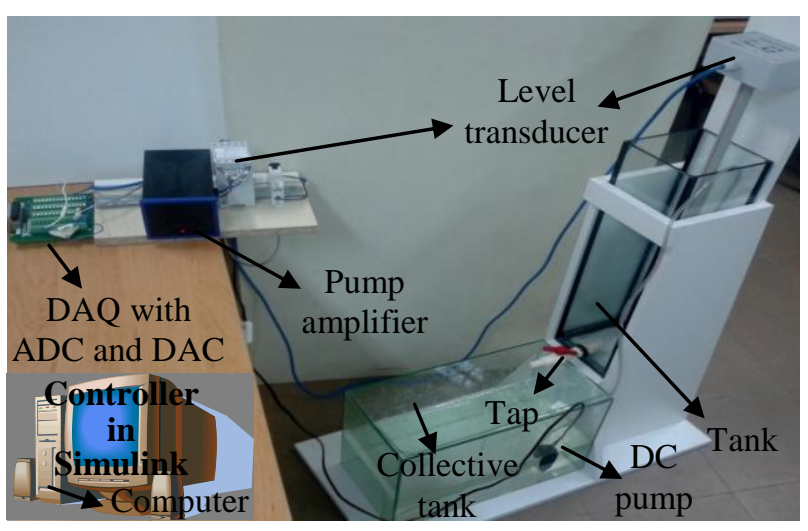

Fig.1. Laboratory-scale pilot plant for level control

A model-free PI-FLC is empirically designed on the basis of standard MFs and rules and the ranges of the error, the derivative of error and the control. The designed closed loop system is shown in Fig. 2, where the

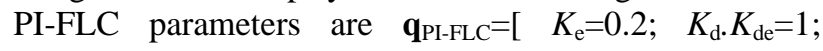
$\left.T_{\mathrm{d}}=(3 \div 10) d t=2 ; K_{\mathrm{du}}=0.5\right]$.

The necessary data for the TSK plant modeling is the plant output $y_{\mathrm{e}}\left(H_{\mathrm{e}}\right)$, recorded in the real time control of level with the designed PI-FLC for typical step changes 
of the closed loop system reference $y_{\mathrm{r}}\left(H_{\mathrm{r}}\right)$.

The TSK plant model accepted is shown in Fig. 3 and is based on the structure, suggested in [21]. It assumes that the plant in the closed loop system operates in three overlapping linear domains for all possible reference changes and disturbances and the dynamics of the local linear plants can be represented by a series connection of two time-lags, the second is common for the three parallel channels, unlike the dynamic models in [21].

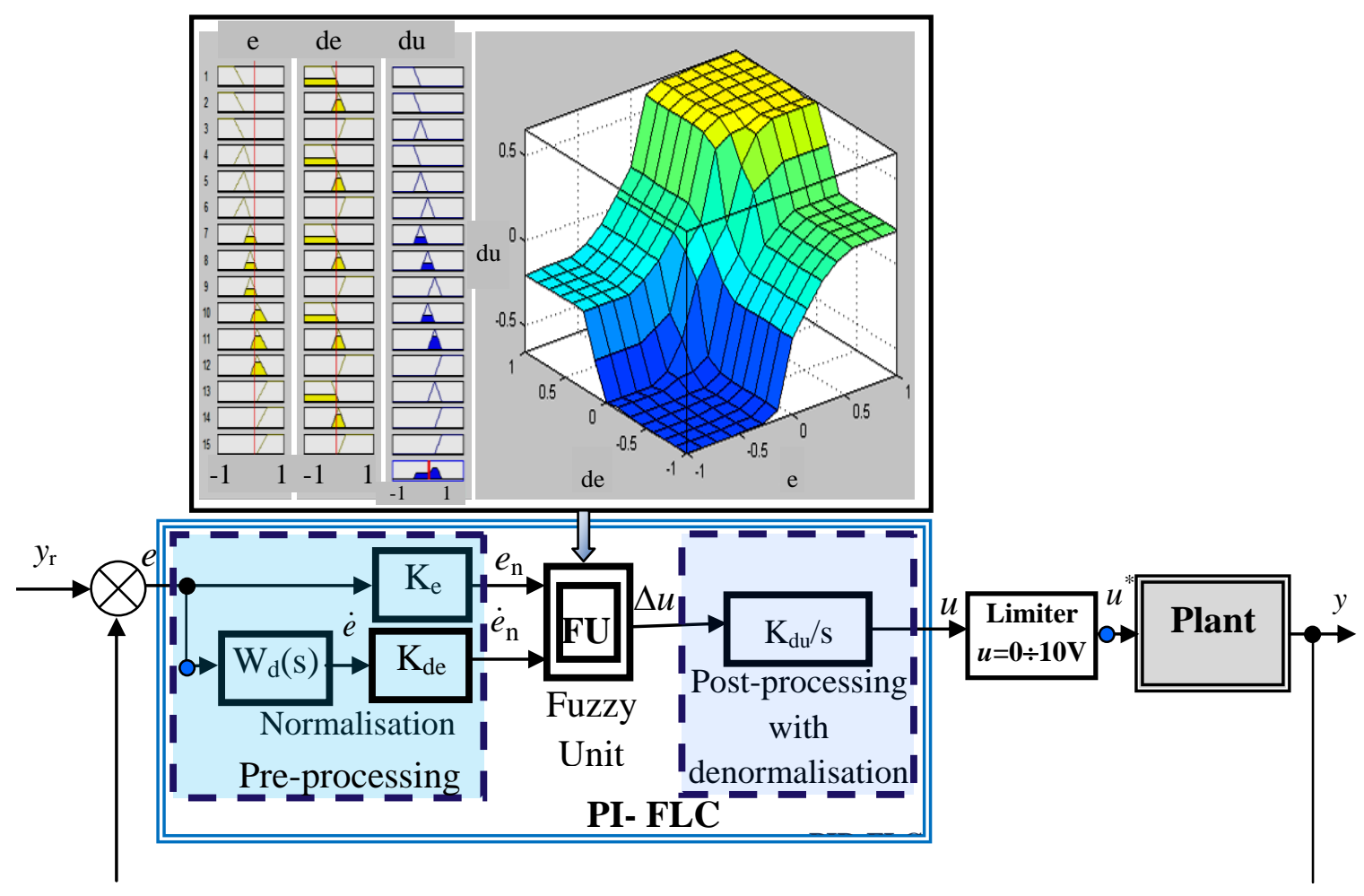

Fig.2. PI-FLC closed loop system

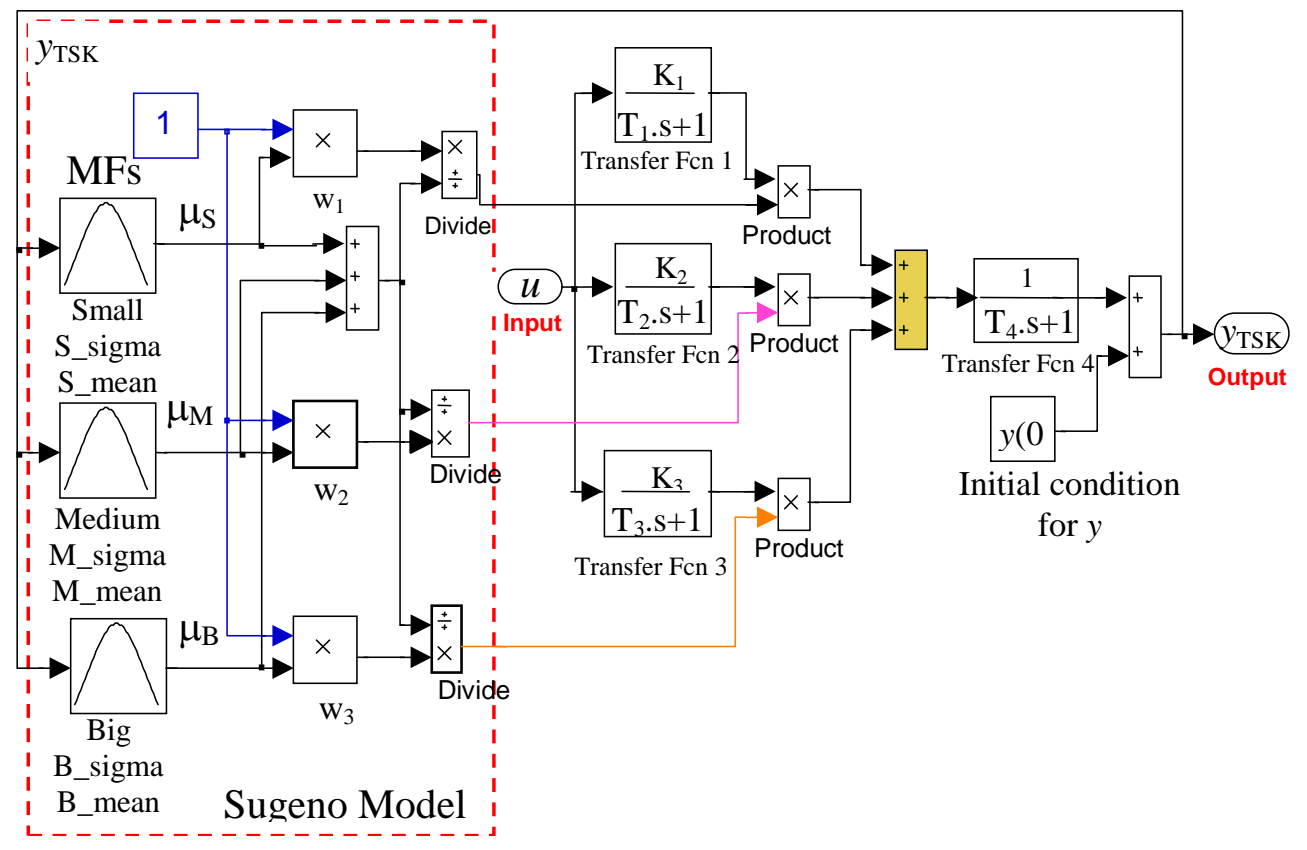

Fig.3. TSK fuzzy plant model

The TSK plant model consists of a zero-order Sugeno Model with fuzzy rules:
If $\left(y_{\mathrm{TSK}}\right.$ is Medium) then $u_{2}$ is 1 If $\left(y_{\mathrm{TSK}}\right.$ is $\left.\mathrm{Big}\right)$ then $u_{3}$ is 1 .

$$
\text { If }\left(y_{\mathrm{TSK}} \text { is Small) then } u_{1} \text { is } 1\right.
$$

The terms Small, Medium and Big, represented by 
Gaussian MFs with parameters (sigma, mean) in Fig. 3, are associated with three expected linearization domains, which are recognized by the TSK plant model output $y_{\mathrm{TSK}}$. The Sugeno Model via the inference mechanism and the weighted average defuzzification serves as a dependent on $y_{\mathrm{TSK}}$ fuzzy blender of the outputs of the parallel linear dynamic models for the linearization domains.

The TSK plant model tuning parameters $\mathbf{q}_{\mathbf{p}}=\left[K_{1} K_{2} K_{3}\right.$ $\begin{array}{llllll}T_{1} & T_{2} & T_{3} & T_{4} & y(0) & \text { S_sigma } \\ \text { S_mean }\end{array}$ B_sigma B_mean] are to be GA optimized via minimization of the integral squared relative error as a fitness function:

$$
F_{\mathrm{p}}=\int\left[E(t) / y_{\mathrm{e}}(t)\right]^{2} \cdot d t
$$

where $E(t)=y_{\mathrm{TSK}}(t)-y_{\mathrm{e}}(t)$ is the relative error, defined as the difference between the outputs of two closed loop systems $-y_{\mathrm{TSK}}(t)$ from the simulated with the TSK plant model, and $y_{\mathrm{e}}(t)$ from the real time control of the real world plant. Both systems have the same empirically tuned PI-FLC and are subjected to the same step reference changes.

Unlike [21] the experimental data $y_{\mathrm{e}}(t)$ used in computing of (2) is recorded from the real time control of the plant in a closed loop system with empirically designed PI-FLC and step-wise changing references with random magnitudes and duration, covering the whole range of the input signals, in order the TSK plant model to learn the real plant nonlinearity while in [21] $y_{\mathrm{e}}(t)$ are the real plant responses from plant identification to more restricted in character step input $u(t)$. If more realistic random input is used, it can still be far from the plant input in real time control in a closed loop system. Besides, such experiments take a long time.

The ready TSK plant model is validated by comparing its response to the real plant response in identification with input signals, different from the used in TSK plant modeling. The real plant and the TSK plant model step responses to the given plant input signals are very close as seen in Fig. 4, where also the TSK plant model optimal parameters are presented. Then the Sugeno model of the validated TSK plant model is completed in a Simulink Fuzzy Logic Controller block as shown in Fig. 5 to make the model compact and convenient for use.

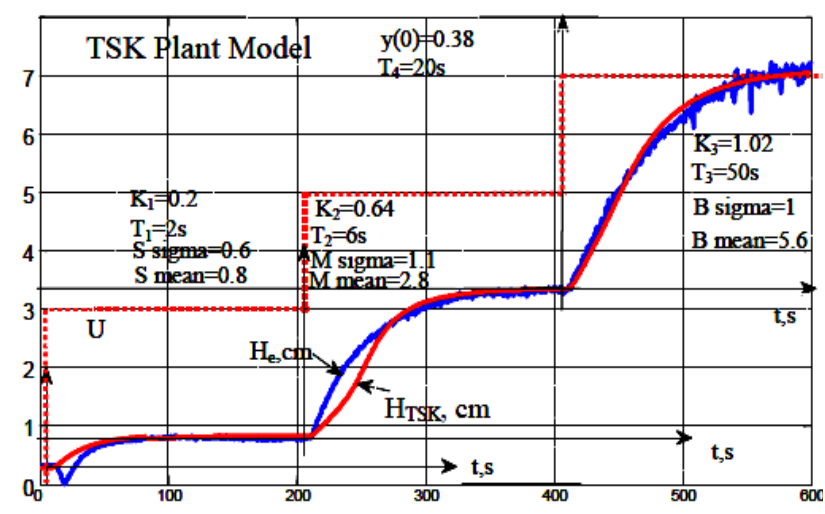

Fig.4. Validation of TSK fuzzy plant model from plant identification input-output data

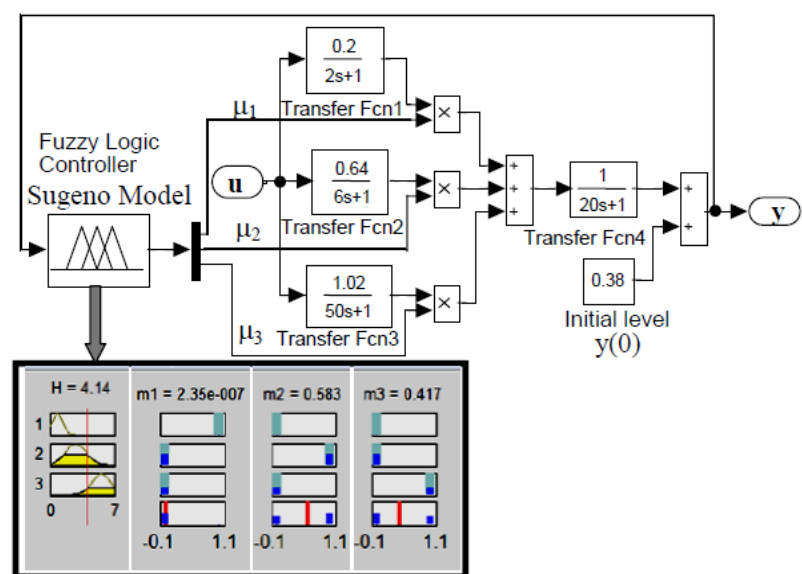

Fig.5. GA optimized TSK plant model with compact FLC block in Simulink for the Sugeno Model

Further this compact and precise TSK plant model is implemented in the Simulink simulation model of the closed loop system employed in the GA optimization of the FLC parameters.

\section{Procedure for OfF-Line GA Optimization of FLC PARAMETERS}

The simulation model of the closed loop system is shown in Fig. 6. It consists of the TSK plant model, controlled by the PI/PID-FLC which parameters have to be off-line GA optimized. For PI/PID-FLC tuning parameters are selected the parameters $\mathbf{q}_{\text {PI/PID-FLC }}$ of the pre- and post-processing $\mathrm{ScF}$ and dynamic components, since their tuning enables by simple means effective shaping of the control surface, equivalent to the more sophisticated techniques of tuning of rules and MFs [24]. The fitness function is (1). The GA optimization is carried out using MATLAB ${ }^{\mathrm{TM}}$ genetic algorithms [20]. The procedure concludes in the following steps.

1. Input data - number of generations $G$, size of population $N$ in a generation, fitness function, initial upper and lower bound for the tuning parameters taken from the empirical tuning of the FLC, end condition (reached number of generations $G$ or minimal value of the fitness function), selection method, crossover rate and method, mutation rate and method

2. Generation of a random initial population of individuals (chromosomes), each chromosome presenting an ordered array of coded tuning parameters.

3. Running of simulations of the closed loop system model for all individuals - PI/PID-FLC tuning parameters, and recording the necessary data used to evaluate the fitness function.

4. Evaluation of the fitness of each individual and ranking of individuals with respect to their fitness value.

5. Check for met end conditions. If "Yes" - the optimal parameters of the PI/PID FLC are found and the procedure terminates, else - step 6 .

6. Selection of survivors - mate parents from the 
population with probabilities proportional to their fitness values - basic methods are roulette wheel selection, uniform, stochastic uniform, normalized geometric selection, remainder, tournament selection.

7. Creation of an offspring (a couple of new chromosomes) by randomly varying individuals - parents applying:

7. a. Crossover over selected parents with a probability equal to the crossover rate. Basic crossover methods are single point - an exchange of genes (a gene is a bit or a parameter) between parents after a given single point (location), multipoint, uniform, scattered.

7. b. Mutation with a probability equal to the mutation rate. Basic mutation methods are Gaussian (Gaussian selection of the bit to be changed), uniform, adaptive feasible (in one or more bits).

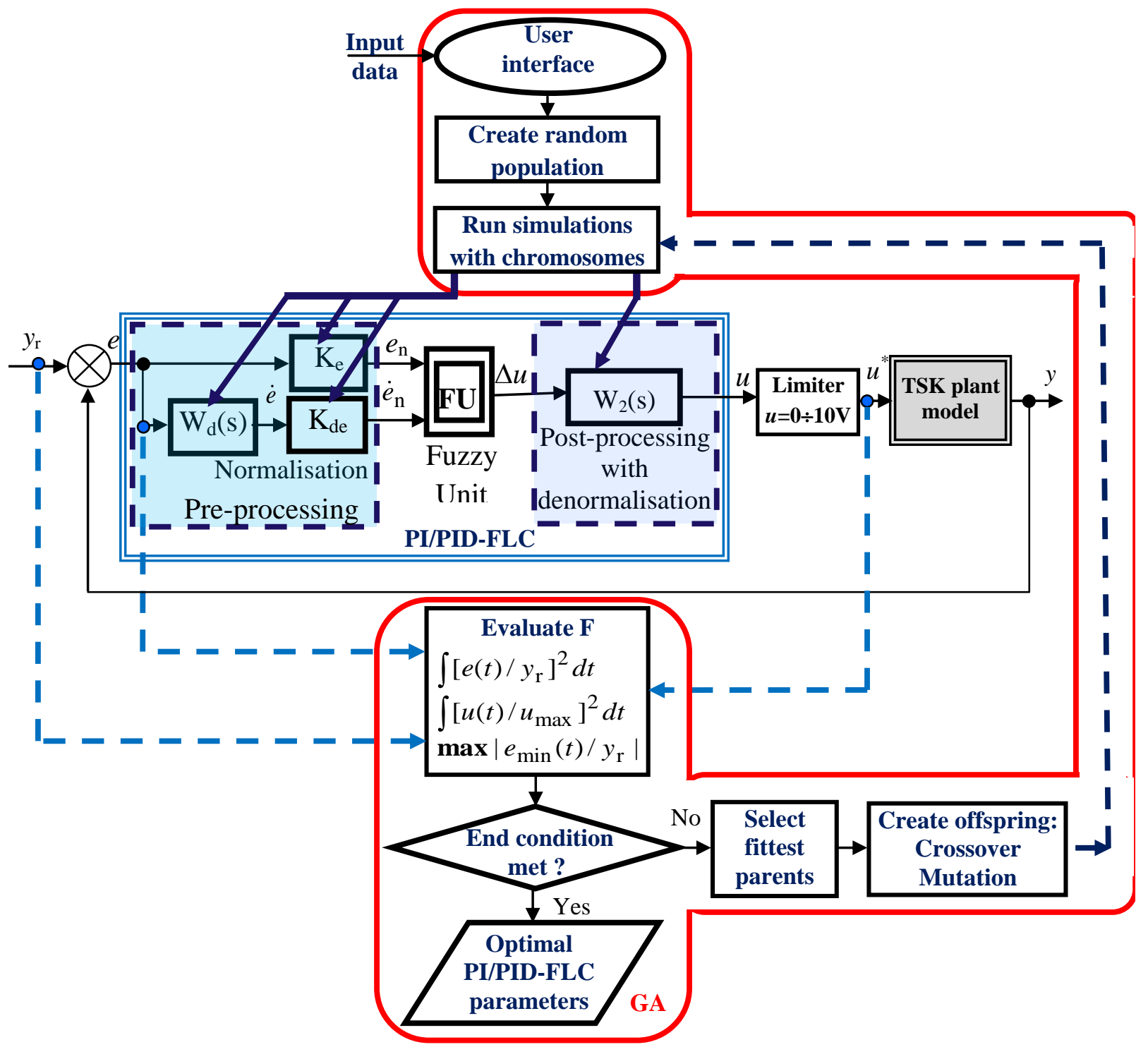

Fig.6. Off-line GA optimization of PI/PID-FLC tuning parameters

8. Running of simulations of the closed loop system model with the new parameters of the offspring, evaluation of the fitness function and acceptance in the new generation if better than the parents, else repeatition from step 6 to step 8 .

Some of the individuals in the current population that have the best fitness can be chosen as elite to pass directly to the next generation.

8. a. Repetition of step 6 to step 8 until the next generation is filled with $N$ members.

8. b. Repetition from step 5 till the accepted end condition is fulfilled.

The fitness function (1) is evaluated after collecting the necessary data as shown in Fig. 6 during the closed loop system model simulation with the GA determined values for the chromosome (set of controller's parameters). Parameter GA optimization employing simulation is a fast and more realistic approach with experiments under full control (no random disturbances or noise effect) and safe for the plant. It also ensures the best solution without restrictions on parameters and signals, their gradual changes, or changes only in steady state for system 
stability and safety reasons. The simulation can consider various inputs - references changes and disturbances with different magnitude and frequency that cover the realistic industrial environment impact range. However, a reliable plant model is required for the whole range of operation conditions, which can be obtained on the basis of intelligent approaches and experimental data.

The procedure is applied for the off-line GA optimization of the parameters $\mathbf{q}_{\mathrm{PI}-\mathrm{FLC}}=\left[K_{\mathrm{e}} K_{\mathrm{d}} K_{\mathrm{de}} T_{\mathrm{d}} K_{\mathrm{du}}\right]$ of the PI-FLC for level control, using Fig. 6 with the developed compact TSK plant model from Fig. 5. The input data are the parameters of the GA - population size $N=20$, number of generations $G=20$, elite -2 , crossover rate -0.8 and method - single point, mutation operator adapt feasible, fitness scaling - rank based, selection - roulette wheel, binary coding. The optimal PI-FLC parameters computed are:

$$
\begin{gathered}
\mathbf{q}_{\text {PI-FLC }}{ }^{\mathbf{o p t}}=\left[K_{\mathrm{e}}=0.12 ;\left(K_{\mathrm{d}} \cdot K_{\mathrm{de}}\right)=3.8 ; T_{\mathrm{d}}=2 ; K_{\mathrm{du}}=0.53\right] . \\
\text { V. REAL TIME CONTROL OF LEVEL AND SYSTEMS } \\
\text { PERFORMANCE ASSESSMENT }
\end{gathered}
$$

\section{REAL Time CONTROL OF LEVEL AND SYSTEMS}

The real time level control is performed using the experimental setup, depicted in Fig. 1, on which the closed loop system in Fig. 2 for PI-FLC with the optimal parameters $\mathbf{q}_{\text {PI-FLC }}{ }^{\text {opt }}$ is completed. The Simulink model of the control algorithm is shown in Fig. 7.

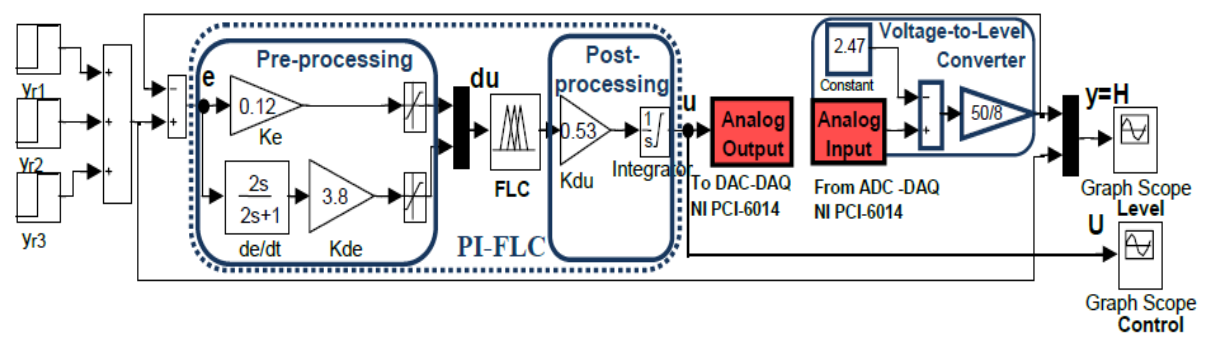

Fig.7. Simulink PI-FLC algorithm for real time control

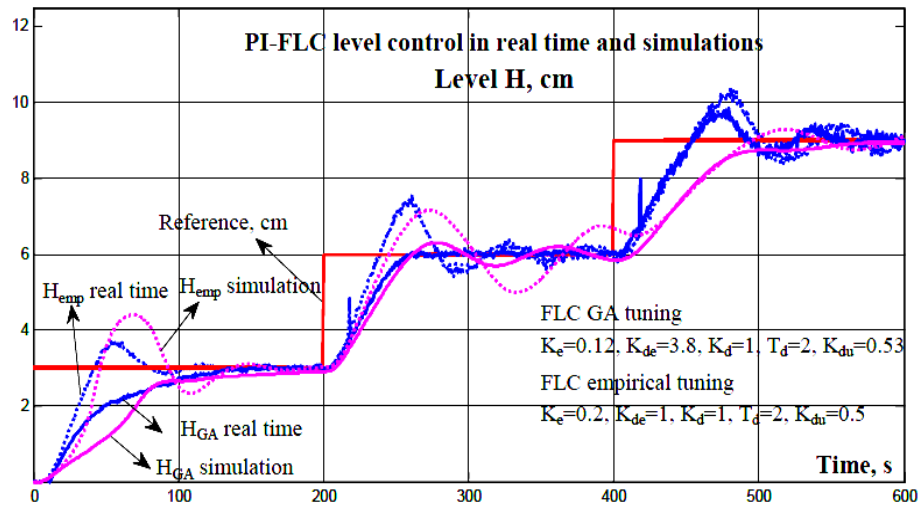

Fig.8. Level from Simulink real time level control and simulations with empirically and GA tuned PI-FLCs

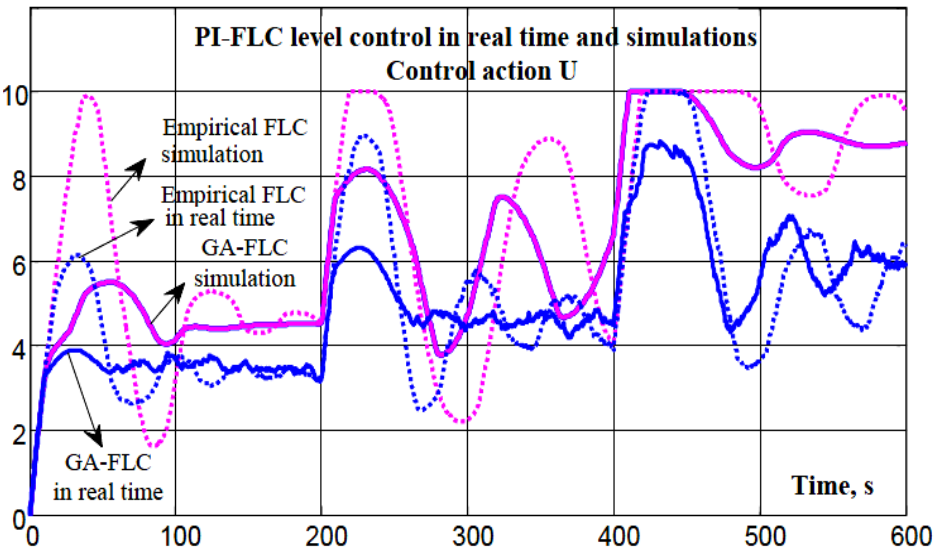

Fig.9. Control action from Simulink real time level control and simulations with empirically and GA tuned PI-FLCs

A number of reference step responses in real time control are studied in two systems - with the GA optimized (Fig. 7) and with the empirical tuned PI-FLC - $\mathbf{q}_{\text {PI-FLC }}=\left[K_{\mathrm{e}}=0.2 ; K_{\mathrm{d}} \cdot K_{\mathrm{de}}=1 ; T_{\mathrm{d}}=2 ; K_{\mathrm{du}}=0.5\right]$, in order to compare their performance indices and assess the expected improvement as a result of the GA optimization. 
The performance is estimated by the settling time $t_{\mathrm{s}}$, the overshoot $\sigma$, the control action maximum $U_{\max }$ and smoothness (magnitude $A$ of the control oscillations) as measures for the control effort and the energy efficiency control oscillations especially with big magnitude $A$ mean low energy efficiency. The reference changes are typical stepwise from different operation points, covering the range of operation of the plant. This allows assessing both the system robustness, expressed as preservation of the system performance indices in different operation points despite the different plant model parameters there, and the impact of the system nonlinearity on the system performance.

The level step responses of the two systems - $H_{\mathrm{emp}}$ with the empirically tuned and $H_{\mathrm{GA}}$ with the GA tuned PI-FLC, from real time control and simulations are overlaid to ease comparison and depicted in Fig. 8. In Fig. 9 the corresponding control actions are presented.

The performance indices of the systems with GA-tuned and empirically-tuned PI-FLC, estimated from real time control and simulations, are presented in Table 1. The system robustness is assessed by the introduced "Robustness index", evaluated from the real time control by the greatest relative deviation of the current performance index value $\left|\mathbf{P}-\mathbf{P}_{\min }\right| \mathbf{P}_{\max }$ from the smallest possible value $\mathrm{P}_{\min }$ (the desired performance value) with respect to the maximal value $P_{\max }$ (the worst index) achieved in both systems with and without GA optimization of the PI-FLC. The comparison of the systems performance indices shows:

Table 1. Comparison of PI-FLC Systems Performance Indices

\begin{tabular}{|c|c|c|c|c|c|c|c|c|c|}
\hline \multirow[t]{2}{*}{ Experiment } & \multirow{2}{*}{$\begin{array}{c}\text { System } \\
\text { performance } \\
\text { index P }\end{array}$} & \multicolumn{4}{|c|}{ Empirical tuned PI-FLC } & \multicolumn{4}{|c|}{ GA optimized PI-FLC } \\
\hline & & $\begin{array}{c}H_{\mathrm{r}}=0-3 \\
\mathrm{~cm}\end{array}$ & $\begin{array}{c}H_{\mathrm{r}}=3-6 \\
\mathrm{~cm}\end{array}$ & $\begin{array}{c}H_{\mathrm{r}}=6-9 \\
\mathrm{~cm}\end{array}$ & $\begin{array}{c}\text { Robustness } \\
\text { index }\end{array}$ & $\begin{array}{c}H_{\mathrm{r}}=0-3 \\
\mathrm{~cm}\end{array}$ & $\begin{array}{c}H_{\mathrm{r}}=3-6 \\
\mathrm{~cm}\end{array}$ & $\begin{array}{c}H_{\mathrm{r}}=6-9 \\
\mathrm{~cm}\end{array}$ & $\begin{array}{c}\text { Robustness } \\
\text { index }\end{array}$ \\
\hline \multirow{4}{*}{$\begin{array}{c}\text { Real time } \\
\text { control }\end{array}$} & $t_{s}, s$ & 120 & 130 & $200=\max$ & 0.7 & 120 & $60=\min$ & 170 & 0.55 \\
\hline & $\sigma, \%$ & 23 & $45=\max$ & 40 & 1 & $0=\min$ & 0 & 23 & 0.51 \\
\hline & $\mathbf{U}_{\max }$ & 6.2 & 8.9 & $10=\mathbf{m a x}$ & 0.62 & $3.8=\min$ & 6.3 & 8.8 & 0.5 \\
\hline & A oscillations & 3.2 & 6 & $6.5=\max$ & 0.6 & $0.5=\min$ & 1.7 & 4.1 & 0.55 \\
\hline \multirow{4}{*}{ Simulations } & $t_{s}, s$ & 140 & 300 & 180 & - & 140 & 200 & 150 & - \\
\hline & $\sigma, \%$ & 45 & 40 & 10 & - & 0 & 10 & 0 & - \\
\hline & $\mathbf{U}_{\max }$ & 9.8 & 10 & 10 & - & 5.5 & 8.1 & 10 & - \\
\hline & A oscillations & 8.3 & 7.8 & 2.5 & - & 1.5 & 4.3 & 1.8 & - \\
\hline
\end{tabular}

- performance indices of simulated and real time step responses are relatively close for each of the systems which confirms the precision of the derived TSK plant model and of the derived on its basis GA optimized PIFLC parameters

- in real time control the GA-tuned system outperforms the empirically tuned system reducing: 1) the overshoot to zero for the first two references and twice for the third reference; 2) the total settling time 1.3 times; 3 ) the total control effort estimated by Umax - 1.33 times; 4) the magnitude of control oscillations - 2.6 times

- in real time control the GA-tuned system has for each performance index $\mathbf{P}$ a smaller robustness index deviation from achieved desired index $\mathrm{P}_{\min }$ with respect to its achieved maximal value $\mathrm{P}_{\max }$ (the worst index for both systems), than the robustness index of the empirically tuned system. This determines the FLC GAoptimization as a tool for making the system more robust - with reference responses less sensitive to the changes of the nonlinear plant parameters in the different operation points, defined by $H_{\mathrm{r}}$.

\section{CONCLUSION AND FUTURE WORK}

The main results of the present research conclude in the following.

1. An easy for engineering applications procedure for off-line GA optimization of the parameters of the preand post-processing of a model-free FLC is suggested. It is demonstrated for the parameter tuning of a PI/PID-FLC with a widely spread structure of a fuzzy unit with normalized inputs - the system error $e$ and derivative-oferror $\dot{e}$, and an output - the rate of control $d u$, where the post-processing is integral for PI-FLC and proportionalplus-integral for PID-FLC respectively. The procedure is based on real time control data from initially empirically designed FLC system, a derived TSK plant model and a suggested multi-objective fitness function, defined on system performance and energy efficiency measures and computed via system simulation.

2. A TSK plant model of an accepted modified structure, consisting of a Sugeno zero order model and parallel branches of dynamic elements, is GA parameter optimized using real plant input-output data from its real time control by an empirically designed FLC in a closed loop system for different references. The TKS plant model is validated on real plant identification data, different from the used in modeling.

3. The developed GA optimization procedure is applied for the real time control in MATLAB ${ }^{\mathrm{TM}}$ of the liquid level in a laboratory-scale pilot tank, equipped with industrial actuator (pump, amplifier) and measuring transducer (pressure difference level transducer and transmitter), in an environment close to the industrial.

The compared performances of the closed loop systems with the empirically tuned FLC and the GA optimized FLC show that GA optimization leads to a significant reduction of settling time, overshoot, control effort and smoothness and contributes to the increase of system energy efficiency and robustness.

Future work will focus on GA optimization of a FLC 
for the real time control of a multivariable plant - the levels in a coupled-tank laboratory system.

\section{ACKNOWLEDGMENT}

The author would like to express her gratitude to the Technical University of Sofia for supporting this research by grant № 152пд0006-08/2015 and to the anonymous reviewers for their professional comments contributing to improvement of the manuscript.

\section{REFERENCES}

[1] T. Neshkov, S. Yordanova, and I. Topalova, Process Control and Production Automation. Sofia: Technical University of Sofia, 2007.

[2] G. Stephanopoulos, Chemical Process Control. An Introduction to Theory and Practice. Prentice Hall, 1984.

[3] F.G. Shinskey, Process Control Systems: Application, Design, Adjustment. $2^{\text {nd }}$ ed., McGraw-Hill, Inc., 1979.

[4] J. Jantzen, Foundations of Fuzzy Control. NY: John Wiley \& Sons, Inc., 2007.

[5] M. Al-H. Basil, M. Fernando, and A. Jiménez, "Fuzzy control for a liquid level system", Proceedings of the $3^{\text {rd }}$ Conference of the European Society for Fuzzy Logic and Technology, Zittau, Germany, September 10-12, 2003.

[6] M. Raduca, E. Raduca, C. Hatiegan, and D. Ungureanu, "Fuzzy controller for adjustment of liquid level in the tank", Annals of the University of Craiova, Mathematics and Computer Science Series, vol. 38(4), 2011, pp. 33-43.

[7] Disha, Mr. P. Pandey, and R. Chugh, "Simulation of water level control in a tank using fuzzy logic", IOSR Journal of Electrical and Electronics Engineering (IOSRJEEE), vol. 2(3), 2012, pp. 9-12.

[8] D. Ahmad, A. Ahmad, V. Redhu, and U. Gupta, "Liquid level control by using fuzzy logic controller", International Journal of Advances in Engineering and Technology, vol. 4(1), 2012, pp. 537-549.

[9] R. Malhotr, and R. Sodhi, "Boiler flow control using PID and fuzzy logic controller", IJCSET, vol. 1(6), 2011, pp. 315-319.

[10] A. Kumar, Rajbir, and Kuldeepak, "Performance comparison of level control with the three, five and nine fuzzy rules based method", International Journal of Advanced Research in Computer Science and Electronics Engineering, vol. 2(7), 2013, pp. 561-566.

[11] R.L. Haupt, and S. El. Haupt, Practical Genetic Algorithms. NY: John Wiley \& Sons, 1998.

[12] B. Kumar, and R. Dhiman, "Optimization of PID controller for liquid level tank system using intelligent techniques", Canadian Journal on Electrical and Electronics Engineering, vol. 2(11), 2011, pp. 531-535.

[13] T. K. Teng, J. S. Shieh, and C. S. Chen, "Genetic algorithms applied in online autotuning PID parameters of a liquid-level control system", $J$. Transactions of the Institute of Measurement and Control, vol. 25(5), 2003, pp. 433-450.

[14] T.T. Erguzel, "Fuzzy controller parameter optimization using genetic algorithm for a real time controlled system", Proceedings of the World Congress on Engineering, vol. 2, London, U.K., 2013.

[15] T.L. Seng, M. Khalid, and R. Yusof, "Tuning of a neurofuzzy controller by genetic algorithms with an application to a coupled-tank liquid-level control system", Int. J. of Engineering Applications on Artificial Intelligence, vol. 11, 1998, pp. 517-529

[16] H. Cho, K. Cho, and B. Wang, "Fuzzy-PID hybrid control: automatic rule generation using genetic algorithms", Fuzzy Sets and Systems, vol. 92, 1997, pp. 305-316.

[17] O.I. Hassanein, A.A. Aly and A.A. Abo-Ismail, "Parameter tuning via genetic algorithm of fuzzy controller for fire tube boiler", IJISA, vol.4, no.4, pp. 9-18, 2012.

[18] Y. Misra and H.R Kamath, "Design algorithm and performance analysis of conventional and fuzzy controller for maintaining the cane level during sugar making process", IJISA, vol.7, no.1, pp. 80-93, 2015. DOI: 10.5815/ijisa.2015.01.08

[19] Fuzzy Logic Toolbox. User's Guide for Use with MATLAB, MathWorks, Inc. 1998.

[20] MATLAB - Genetic Algorithm and Direct Search Toolbox. User's Guide, MathWorks, Inc., 2004.

[21] S. Yordanova, "Intelligent approaches for linear controllers tuning with application to temperature control", Journal of Intelligent and Fuzzy Systems, IOS Press, NL, vol. 27(6), 2014, pp. 2809-2820.

[22] S. Yordanova, and Y. Sivchev, "Design and tuning of parallel distributed compensation-based fuzzy logic controller for temperature", Journal of Automation and Control, vol. 2( 3), 2014, pp. 79-85.

[23] S. Yordanova, and A. Georgieva, "Genetic algorithm based optimization of fuzzy controllers tuning in level control", $J$. Electrotechnica and Electronica E+E, vol. 48(9-10), 2013, pp. 45-51.

[24] S. Yordanova, Design of Fuzzy Logic Controllers for Robust Process Control. Sofia: KING, 2011.

\section{Authors' Profiles}

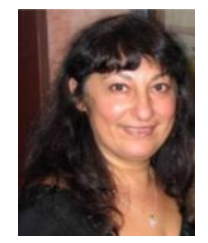

Snejana Yordanova was born in Sofia, Bulgaria in 1953. She received her M.Sc. degree in 1978 and Ph.D in 1986 both in process control from the Technical University of Sofia, Bulgaria.

She joined the Process Control department of the Faculty of Automation at the Technical University of Sofia in 1980. From 1998 she is an Associate Professor and from 2011 till now - a full time Professor.

Her research and academic interests and experience are application of robust, fuzzy logic, neural network and genetic algorithms approaches for modelling and control of plants mainly from ecology, power engineering, air-conditioning, and oil-refining. She has published one monographical book [22], one book chapter, and is the author and co-author of 15 texbooks and over 120 papers most of which in prestigeous reviewed journals and some with impact factor (IEEE TFS, JIFS etc.). She has $5 \mathrm{Ph} . \mathrm{D}$ students and over 30 industrial and academic projects. She is in the editorial board of a number of journals and in the organizing committee of international conferences. She is reviewing for journals (IEEE TFS, APIN, J.Control Sc\&Eng, etc.) and conferences and is a member of several automation societies - WSEAS, Union of Automatica and Informatics, etc. Her plenary paper "Design of robust fuzzy logic controllers for complex non-linear processes with time delay" at the $8^{\text {th }}$ WSEAS Int. Conf. on Artificial Intelligence, Knowledge Engineering and Data Bases - AIKED'09, Cambridge, UK, 21-23 Feb., 2009 was nominated as the Best paper Award. 